\title{
Study on the Alternatives to Reduce High-Frequency Noise from Invasive Recordings of Atrial Fibrillation
}

\author{
Miguel Martínez-Iniesta ${ }^{1}$, Juan Ródenas ${ }^{1}$, Raúl Alcaraz ${ }^{1}$, José J Rieta ${ }^{2}$ \\ ${ }^{1}$ Research Group in Electronic, Biomedical and Telecomm. Eng., Univ. of Castilla-La Mancha, Spain \\ ${ }^{2}$ BioMIT.org, Electronic Engineering Department, Universitat Politecnica de Valencia, Spain
}

\begin{abstract}
Catheter ablation (CA) is nowadays the first-line therapy for the treatment of atrial fibrillation $(A F)$. This approach relies heavily on cardiac mapping systems, which provide intra-cardiac electrograms (EGMs) to study the heart's electrical activity. To get precise clinical information from these signals, the elimination of noise and nuisance interferences, as well as the preservation of their original waveform, are key aspects to treat carefully. However, how they should be preprocessed to remove successfully high-frequency noise has only received limited attention to date. Hence, in this study the most commonly bandpass filtering applied to this recording is compared with two advanced denoising methods, based on Wavelet Transform (WT) and Empirical Mode Decomposition (EMD), on a set of 150 synthesized bipolar EGMs with different levels of noise. The resulting signals have been contrasted with the original ones in terms of a signed correlation index (SCI), computed both from time and frequency domains. Thus, notably more significant alterations in the original waveform have been observed for the regular filtering than for the WT-based approach. As a conclusion, this method is a more interesting option than the regular filtering to preserve the morphology of the bipolar EGM and obtain more accurate electrophysiological information.
\end{abstract}

\section{Introduction}

Atrial fibrillation (AF) is becoming a serious cardiovascular problem worldwide [1]. It is a very common supraventricular arrhythmia in clinical practice, affecting approximately $2 \%$ of the general adult population [2]. Moreover, its incidence and prevalence increase with age, such that about $15 \%$ of people older than 80 years suffers from this arrhythmia [2]. However, the underlying pathophysiological mechanisms triggering and maintaining this disease are still not fully understood [3]. Thus, the atrial activity analysis has been identified by many experts as a priority to improve our prevailing knowledge about AF [4].
In a broad variety of clinical studies the atrial information is obtained from the patient's thorax. Indeed, the surface electrocardiogram (ECG) is currently the most used signal for the diagnosis of cardiovascular disorders [5]. However, the intra-cardiac electrogram (EGM) has proven a higher ability to reflect the electric status of the myocardium, thus being today the hallmark of cardiac electrophysiology [6]. Although two different kinds of signals are widely registered during common electrophysiology procedures, the bipolar EGM is receiving increased attention compared to the unipolar signal [6]. This EGM is obtained by subtracting the recordings captured by two electrodes nearly located in a specific area, thus mainly reflecting information about its local activity [7].

However, such as the surface ECG, the bipolar EGM is also usually disturbed by noise and nuisance interferences. Hence, its successful and accurate preprocessing plays a key role for further analysis [8]. To this respect, some previous works have identified a minimum signal-to-noise ratio (SNR) of $20 \mathrm{~dB}$ as a requirement to discern small changes in the EGM [8]. Unfortunately, this aspect has not received much attention in the literature and, usually, the bipolar signal is severely filtered between 30 and $300 \mathrm{~Hz}$ during its acquisition, even when it has been proven that this preprocessing disturbs notably its amplitude, timing and morphology [7]. Within this context, the main goal of the present work is to analyze if two advanced denoising methods, proposed to deal with the surface ECG, are also able to obtain cleaner and less morphologically altered bipolar EGMs than the described filtering.

\section{Materials}

To validate the proposed denoising algorithms, a set of synthesized bipolar EGMs was used, because these signals allow to quantify accurately the achieved noise reduction and how the original waveform is preserved. Thus, using the methodology proposed by Oesterlin et al. [9], 150 realistic, completely clean and 10 second-length bipolar EGMs were generated. To obtain noisy recordings, a highfrequency noise was then added to the signals. This per- 


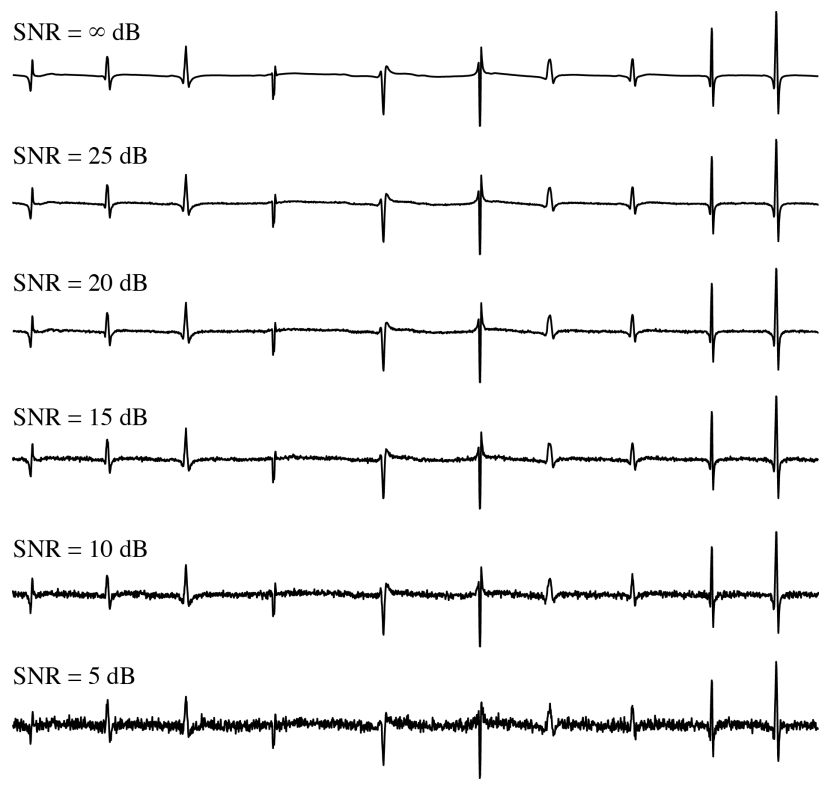

Figure 1. Example of a synthesized bipolar EGM with different levels of noise.

turbation was generated following the approach described in [10] and normalized in amplitude to synthesize EGMs with values of SNR of $25,20,15,10$ and $5 \mathrm{~dB}$. As an example, Fig. 1 shows a typical synthetic bipolar EGM with different levels of noise. Note that the clean signal will be referred to as $x(n)$ throughout the text .

\section{Methods}

The next subsections describe the regular filtering applied to the bipolar EGM during its acquisition and how two effective methods reducing high-frequency noise from the ECG are adapted to work with this intra-cardiac recording. The proposed algorithms are based on transforming the original signal by means of Wavelet transform (WT) and Empirical Mode Decomposition (EMD).

\subsection{Regular filtering of the bipolar EGM}

As a trade-off between noise reduction and minimum distortion, the bipolar EGM is often low-pass filtered with a cut-off frequency around $300 \mathrm{~Hz}$ [7]. Additionally, with the aim of reducing the far-field ventricular contamination, this signal is also high-pass filtered with a cut-off frequency about $30 \mathrm{~Hz}$ [11]. However, this last approach also reduces considerably the main atrial component, which is usually located between 3 and $12 \mathrm{~Hz}$. Clearly, this filtering distorts the EGM morphology and reduces its amplitude, thus making the recording more complex and fractionated [11]. Nonetheless, a variety of clinical studies have proven that useful information still remains in the sig- nal [7]. To reproduce this regular filtering, a second-order bidirectional Butterworth filter with a bandwidth between 30 and $300 \mathrm{~Hz}$ was used.

\subsection{WT-based denoising}

WT has been broadly used for the ECG denoising due to its ability to deal with non-stationary signals, which contain sharp spikes and discontinuous intervals [12]. This transformation decomposes the signal at different time and frequency scales, which are then rescaled and thresholded to remove the noise [13]. The discrete WT (DWT) has been widely used in biomedical applications, because its implementation can be easily reached through a bank of low-pass and high-pass finite impulse response filters followed by decimation stages [13]. Therefore, this tool was here used with a second-order Coiflet function as wavelet mother to decompose the bipolar EGM into 8 levels.

The obtained wavelet coefficients were rescaled by the standard deviation of the noise, which was estimated from median of the highest frequency level by using the approach described in [14]. Thereafter, they were softly thresholded making use of a cut-off point obtained from the Stein's unbiased risk estimate [14] and, finally, were used to reconstruct the denoised signal.

\section{3. $\quad$ EMD-based denoising}

Compared to the WT, the main advantage of EMD is that it does not need any a priori information to decompose a signal. More precisely, this tool breaks down the original signal into a sum of oscillatory data, called intrinsic mode functions (IMFs), ranging from higher to lower frequencies. Therefore, EMD can also be considered as a filter bank, but without a predefined cut-off frequency [15]. Thus, EMD is an interesting tool to deal with nonlinear and non-stationary signals and, in fact, it has been widely used for the denoising of many physiological signals including, of course, cardiovascular recordings such as the ECG [16].

Considering that the IMFs from the lower levels present higher frequency components and viceversa, some previous works have shown that high-frequency noise can be successfully removed from the ECG by discarding the first IMFs [17]. Consequently, to denoise the bipolar EGM signal, the two first IMFs were here thrown back.

\subsection{Performance assessment}

To assess how the noise is reduced and the EGM morphology is preserved by the proposed denoising algorithms, the resulting signal, referred to as $\hat{x}(n)$, was compared with the original one in terms of correlation. For a broader comparison, in addition to the correlation between 
$x(n)$ and $\hat{x}(n)$, their frequency contents were also analyzed. Thus, the spectral transformation of these signals was reached through a Welch periodogram with a resolution of $0.1 \mathrm{~Hz}$.

Because the classical Pearson's correlation index does not take into consideration the amplitude differences between signals, a signed correlation index (SCI) was used for a more accurate morphological comparison [18]. This metric can be defined for a $L$ sample-length signal as

$$
\mathrm{SCI}(x(n), \hat{x}(n))=\frac{1}{L} \sum_{k=1}^{L} x(k) \otimes \hat{x}(k),
$$

where $\otimes$ is computed as

$$
x(n) \otimes \hat{x}(n)=\left\{\begin{aligned}
1 & \text { if }|x(n)-\hat{x}(n)| \leq \xi, \\
-1 & \text { if }|x(n)-\hat{x}(n)|>\xi .
\end{aligned}\right.
$$

An experimental value of $5 \%$ of the standard deviation of $x(n)$ was selected as the threshold $\xi$.

\section{Results}

Average values of SCI obtained both from time and frequency domains are summarized in Table 1. As expected, the three methods provided more altered time morphology and frequency content as the SNR decreased. Nonetheless, the WT-based algorithm reported the cleanest and most unaltered EGMs compared to the remaining approaches. It is interesting to note that its performance was notably better than the regular filtering for any SNR, but especially for moderate and high levels of high-frequency noise. Similarly, the EMD-based algorithm also provided poorer results than the WT-based method, although notably better than the regular filtering. These findings can be graphically observed for a typical EGM in Fig. 2.

\section{Discussion}

The development of new algorithms able to reduce highfrequency noise in the bipolar EGM but, at the same time, preserving its original waveform, is still an unsolved challenge. Indeed, the obtained results have proven that the regular filtering is not sufficiently effective to remove moderate levels of noise, moreover disturbing both time morphology and frequency distribution of the signal. For instance, even for the most favorable case of $25 \mathrm{~dB}$ in SNR, the regular filtering have only reported a time correlation of about $50 \%$. This outcome could be justified by the elimination of the significant and high-amplitude atrial components located between 1 and $30 \mathrm{~Hz}$.

Contrarily, the proposed WT- and EMD-based algorithms have been able to obtain bipolar EGMs with less altered features of time morphology and frequency content, even for the highest level of noise (i.e. a SNR value
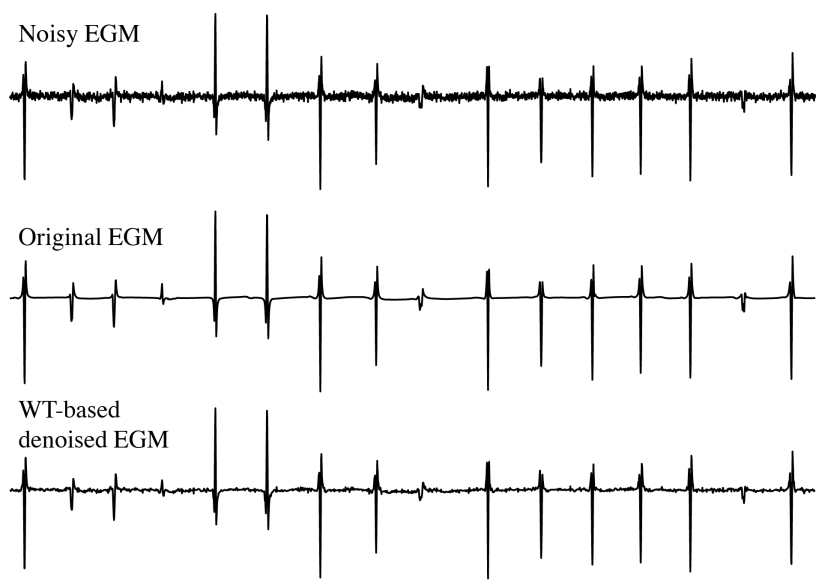

EMD-based

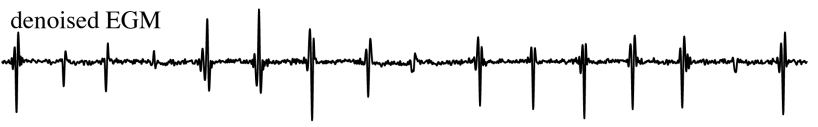

Regular filtered

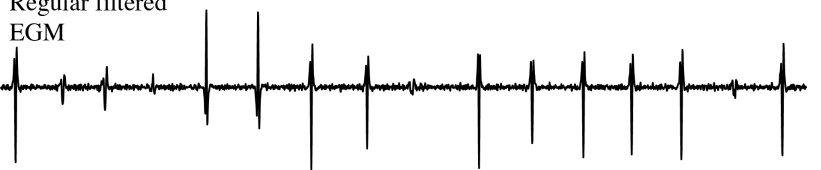

Figure 2. Comparison of denoised signals from the proposed algorithms for a typical bipolar EGM with an original SNR of $15 \mathrm{~dB}$.

of $5 \mathrm{~dB}$ ). Nonetheless, it has to be remarked that the WTbased algorithm provided the cleanest and least distorted signals. Thus, for high levels of noise $(10 \mathrm{~dB})$, values of SCI from time and frequency domains were higher than $84 \%$ and $99 \%$, respectively. Additionally, although the EMD-based approach has improved notably the regular filtering performance, it provided noisier signals than the WT-based algorithms. This result could be explained by the fact that low frequency IMFs can still contain information from the atrial activations, which is discarded as noise. Another relevant disadvantage of the EMD-based approach is its high computational load compared to the WT-based method.

From a clinical point of view, reducing high-frequency noise and preserving the bipolar EGM morphology is mandatory, because in other case the link between the morphological features in the signal and the underlying electrophysiological mechanisms could be obscured. Moreover, if the EGM is too aggressively preprocessed, some interesting information may be lost and further analysis could be distorted. Thus, only when preprocessing is able to preserve the original morphology of the EGM, realistic and reliable analyses can be reached. Hence, the obtained results shown the need of developing more accurate preprocessing methods for the bipolar EGM. In this way, less conflicting and more conclusive results than to date could be reached from electrophysiological studies. 
Table 1. Comparison of the denoising algorithms in terms of SCI both from time and frequency domains.

\begin{tabular}{llccccc}
\hline \hline \multirow{2}{*}{ Method } & & \multicolumn{5}{c}{ SNR (dB) } \\
\cline { 3 - 7 } SCI (\%) & $\mathbf{2 5}$ & $\mathbf{2 0}$ & $\mathbf{1 5}$ & $\mathbf{1 0}$ & $\mathbf{5}$ \\
\hline \multirow{2}{*}{ WT-based } & Time & $99.5 \pm 0.4$ & $97.9 \pm 1.4$ & $93.5 \pm 3.8$ & $83.9 \pm 8.8$ & $70.0 \pm 13.1$ \\
& Freq. & $99.9 \pm 0.02$ & $99.9 \pm 0.02$ & $99.9 \pm 0.03$ & $99.5 \pm 0.5$ & $96.9 \pm 2.3$ \\
\hline \multirow{2}{*}{ Regular Filt. } & Time & $92.7 \pm 2.0$ & $92.0 \pm 3.2$ & $81.6 \pm 9.6$ & $60.7 \pm 13.8$ & $38.9 \pm 11.5$ \\
& Freq. & $94.2 \pm 3.9$ & $95.4 \pm 3.3$ & $96.8 \pm 2.7$ & $96.4 \pm 2.3$ & $90.8 \pm 4.9$ \\
\cline { 3 - 7 } & Freq. & $50.1 \pm 18.2$ & $48.8 \pm 18.0$ & $45.0 \pm 17.5$ & $37.8 \pm 15.3$ & $28.5 \pm 11.5$ \\
& & $93.7 \pm 1.1$ & $93.6 \pm 1.1$ & $93.6 \pm 1.2$ & $92.1 \pm 2.6$ & $84.1 \pm 6.9$ \\
\hline \hline
\end{tabular}

\section{Conclusions}

The proposed denoising algorithm based on Wavelet transform has provided a higher ability to reduce highfrequency noise and to preserve the morphology of the bipolar EGM than regular filtering. Consequently, to obtain more accurate further electrophysiological studies, this approach should replace the habitual way to denoise bipolar electrograms.

\section{Acknowledgements}

Research supported by grants TEC2014-52250-R and DPI2017-83952-C3 MINECO/AEI/FEDER, UE, and PEII-2014-028-P from Junta de Comunidades de CastillaLa Mancha.

\section{References}

[1] Potter BJ, Le Lorier J. Taking the pulse of atrial fibrillation. Lancet Jul 2015;386(9989):113-5.

[2] Zoni-Berisso M, Lercari F, Carazza T, Domenicucci S. Epidemiology of atrial fibrillation: European perspective. Clin Epidemiol 2014;6:213-20.

[3] Schotten U, Dobrev D, Platonov PG, Kottkamp H, Hindricks G. Current controversies in determining the main mechanisms of atrial fibrillation. J Intern Med May 2016; 279(5):428-38.

[4] Heijman J, Algalarrondo V, Voigt N, Melka J, Wehrens XHT, Dobrev D, Nattel S. The value of basic research insights into atrial fibrillation mechanisms as a guide to therapeutic innovation: a critical analysis. Cardiovasc Res Apr 2016;109(4):467-79.

[5] Tso C, Currie GM, Gilmore D, Kiat H. Electrocardiography: A technologist's guide to interpretation. J Nucl Med Technol Dec 2015;43(4):247-52.

[6] de Bakker JMT, Wittkampf FHM. The pathophysiologic basis of fractionated and complex electrograms and the impact of recording techniques on their detection and interpretation. Circulation Arrhythmia and Electrophysiology Apr 2010;3(2):204-13.

[7] Stevenson WG, Soejima K. Recording techniques for clinical electrophysiology. J Cardiovasc Electrophysiol Sep 2005;16(9):1017-22.
[8] Venkatachalam KL, Herbrandson JE, Asirvatham SJ. Signals and signal processing for the electrophysiologist: part i: electrogram acquisition. Circ Arrhythm Electrophysiol Dec 2011;4(6):965-73.

[9] Oesterlein TG, Lenis G, Rudolph DT, Luik A, Verma B, Schmitt C, Dössel O. Removing ventricular far-field signals in intracardiac electrograms during stable atrial tachycardia using the periodic component analysis. J Electrocardiol 2015;48(2):171-80.

[10] Corino VDA, Rivolta MW, Sassi R, Lombardi F, Mainardi LT. Ventricular activity cancellation in electrograms during atrial fibrillation with constraints on residuals' power. Med Eng Phys Dec 2013;35(12):1770-7.

[11] Issa ZF, Miller JW, Zipes DP. Clinical arrhythmology and electrophysiology: a comparison to Braunwald's heart disease. (Chapter 4). 2nd edition. Elsevier, 2012.

[12] Singh BN, Tiwari AK. Optimal selection of wavelet basis function applied to ECG signal denoising. Digital Signal Processing 2006;16(275-287).

[13] Addison PS. Wavelet transforms and the ECG: a review. Physiol Meas Oct 2005;26(5):R155-99.

[14] Donoho D, Johnstone I. Ideal spatial adaptation by wavelet shrinkage. Biometrika 1994;81:425-455.

[15] Flandrin P, Rilling G, Goncalves P. Empirical mode decomposition as a filter bank. IEEE Signal Processing Letters 2004;11(112-114).

[16] Blanco-Velasco M, Weng B, Barner KE. ECG signal denoising and baseline wander correction based on the empirical mode decomposition. Comput Biol Med Jan 2008; 38(1):1-13.

[17] Chang KM. Ensemble empirical mode decomposition for high frequency ECG noise reduction. Biomed Tech Berl Aug 2010;55(4):193-201.

[18] Lian J, Garner G, Muessing D, Lang V. A simple method to quantify the morphological similarity between signals. Signal Processing 2010;90:684-688.

Address for correspondence:

Miguel Martínez Iniesta

E.I. Industriales, Campus Universitario, 02071, Albacete, Spain.

Phone: +34-967-599-200 Ext. 2555

Fax: +34-967-599-224

e-mail: miguel.martinez@uclm.es 